\title{
INVARIANT MEASURES DEFINED BY DIFFERENTIAL EQUATIONS
}

\section{MARKUS ${ }^{1}$}

1. Introduction. If the real differential system $S: d x / d t=f(x, y)$, $d y / d t=g(x, y)$, with $f(x, y), g(x, y)$ in class $C^{(1)}$ in an open plane set $R$, is exact, $\partial f / \partial x+\partial g / \partial y \equiv 0$, then by Liouville's theorem the flow along the trajectories preserves Lebesgue measure. If $S$ is not exact but possesses an integrating factor $\mu(x, y) \in C^{(1)}$ in $R$ such that $\partial(\mu f) / \partial x+\partial(\mu g) / \partial y \equiv 0$, then using the relation

$$
\frac{d}{d t} \iint_{x_{t}} \mu(x, y) d m=\iint_{x_{0}}\left[\frac{\partial(\mu f)}{\partial x}+\frac{\partial(\mu g)}{\partial y}\right] \frac{\partial(x, y)}{\partial\left(x_{0}, y_{0}\right)} d m
$$

where $\chi_{t}$ is the $t$-translate of $\chi_{0} \in \mathcal{L}$, the $\sigma$-ring of Lebesgue measurable sets with Lebesgue measure $m$, Poincaré [8]2 defined an invariant measure $\mu$ on $\mathcal{L}$ by

$$
\mu(\chi)=\iint_{\chi} \mu(x, y) d m \quad \text { for } \chi \in \mathcal{L} .
$$

If $\mu(x, y)>0$ in $R$, then $\mu$-measure and $m$-measure are each ababsolutely continuous with respect to the other, that is, they have the same null sets. The relation (2) is the Radon-Nikodym theorem greatly strengthened in that $\mu(x, y)$ is not only measurable but continuously differentiable. This improvement is possible, of course, only because of the special differential origin of the measure $\mu$.

The invariant measures of Kryloff and Bogoliouboff [6] for flows on compact metric spaces and those of Ulam and Oxtoby [9] and later of Halmos [1] for discrete groups on locally compact metric spaces are not absolutely continuous with respect to Lebesgue measure and no attention is paid in these results to special differential properties.

In the neighborhood of a noncritical point $\left(f^{2}+g^{2}>0\right)$ there exists a local integral function $\psi(x, y)$ for $\delta$ and a corresponding local integrating factor

$$
\mu(x, y)=\left[\left(\psi_{x}^{2}+\psi_{y}^{2}\right) /\left(f^{2}+g^{2}\right)\right]^{1 / 2} .
$$

Received by the editors April 14, 1952.

${ }^{1}$ Work done at Harvard University under Project N5ori-07634 with the Office of Naval Research.

2 Numbers appearing in brackets refer to the bibliography at end of paper. 
However, in the large there generally does not exist a global integral $\psi(x, y) \in C^{(1)}$ with nonvanishing gradient, even if $S$ is $C^{(\infty)}$ and noncritical in $R$. But by piecing together local integrals one can construct $[4 ; 7]$, for a large class of important differential systems, a principal global integral, that is, $\psi(x, y) \in C^{(1)}$, which is constant along each trajectory of $S$ but not constant on any open set. In fact if $S$ is of class $C^{(\alpha)}, \alpha=1,2, \cdots, \infty$, then one can choose $\psi(x, y)$ $\in C^{(\alpha)}$. The gradient of a principal integral of $\delta$ has a set of zeros which is closed and nowhere dense in $R$ and these properties are sufficient to complete the usual theory [5, p. 312] of functional dependence of the integrals of $S$.

In this note we show that even if $\delta$ is $C^{(\infty)}$ and noncritical in the plane, with a principal global integral $\psi(x, y) \in C^{(\infty)}$, the resulting $\mu$-measure may vanish on sets of positive Lebesgue measure. In this case $\mu$-measure and $m$-measure are not equivalent and, in fact, $\mu$ is not complete on $\mathcal{L}$. However if both $\mathcal{S}$ and $\psi(x, y)$ are in $C^{(A)}$ (analytic in two real variables), then $\mu(\chi)=0$ if and only if $m(\chi)=0$ for $\chi \in \mathcal{L}$ and the measures are equivalent.

2. Invariant measures of $C^{(\infty)}$ and $C^{(1)}$ differential systems. Let $z$ be a compact, nowhere dense (and thus with null interior) linear point set with positive Lebesgue measure. One such set [3, pp. 119, 164], similar to the Cantor discontinuum, consists of the closure of the end points of a countable number of disjoint open subintervals $I_{n} \subset[0,1]$. Now define

$$
\begin{aligned}
& \mu(x, y)=0 \text { if } x \in z, \\
& \mu(x, y)=\exp \left[-x^{-2}\right] \text { if } x<0, \\
& \mu(x, y)=\exp \left[-(x-1)^{-2}\right] \text { if } x>1, \\
& \mu(x, y)=\exp -\left[\Delta_{n}^{2} / 4-\left(\Delta_{n} / 2-d(x)\right)^{2}\right]^{-1} \text { if } x \in I_{n},
\end{aligned}
$$

where $\Delta_{n}$ is the length of $I_{n}$ and $d(x)$ is the distance from $x$ to the compact set $z$. A routine calculation shows that $\mu(x, y) \geqq 0$ is of class $C^{(\infty)}$ everywhere in the plane and the set $Z$ on which $\mu(x, y)=0$ has infinite Lebesgue measure, and is invariant under the flow of $S$.

EXAmple. Let $S: \dot{x}=\mu(x, y), \dot{y}=-2 \mu_{x} y+1$, where $\mu(x, y)$ is the function constructed above. Here $\delta$ is of class $C^{(\infty)}$ in the plane and has no critical points since $\mu(x, y)=0$ implies $-2 \mu_{x} y+1=1$. There is a principal global integral $\psi(x, y) \in C^{(\infty)}, \psi(x, y)=y \mu^{2}-\int_{0}^{x} \mu(\xi, y) d \xi$, with a corresponding integrating factor $\mu(x, y)$. The $\mu$-measure is not complete on $\mathcal{L}$ since $\mu(Z)=0$ even though $m(Z)=\infty$ and thus there exists [2, p. 70] a subset $W \subset Z$ but $W \in \mathcal{L}$. 
TheOREM. Let $S: \dot{x}=f(x, y), \dot{y}=g(x, y)$ be a noncritical analytic differential system in the plane and therein possess an analytic principal integral $\psi(x, y)$. Then the $\mu$-measure defined by (2) and (3) is equivalent to Lebesgue measure.

Proof. A necessary and sufficient condition that the $\mu$-measure corresponding to a principal integral be equivalent to $m$-measure is that the variety $Z$ of zeros of the integrating factor $\mu(x, y)$ be a Lebesgue null set. But since $\nabla \psi$ is analytic and $Z$ is closed and nowhere dense in the plane, one can easily show that, with countably many exceptions, the lines $y=$ constant intersect $Z$ in a countable point set. Thus $m(Z)=0$ by Fubini's theorem and $\mu(x, y) \in C^{(\Delta)}$ a.e.

\section{BIBLIOGRAPHY}

1. P. Halmos, Invariant measures, Ann. of Math. vol. 48 (1947) pp. 735-754.

2. - Measure theory, New York, 1950.

3. E. Hobson, The theory of functions of a real variable, Cambridge, 1921.

4. E. Kamke, Über die partielle Differentialgleichungen $f z_{x}+g z_{y}=h$, Math. Zeit. vol. 41 (1936) pp. 56-66 and Math. Zeit. vol. 42 (1936) pp. 287-300.

5. - Differentialgleichungen reeller Funktionen, New York, 1947.

6. N. Kryloff and N. Bogoliouboff, La theorie gentrale de la mesure, Ann. of Math. (1937) p. 65.

7. L. Markus, Global structure of ordinary differential equations in the plane, to appear soon. 1892.

8. H. Poincaré, Méthodes nouvelles de la mécanique celeste, vol. 3, chap. 26, Paris,

9. S. Ulam and J. Oxtoby, On the existence of a measure invariant under a transformation, Ann. of Math. vol. 40 (1939) pp. 560-566.

Harvard University 